\title{
Violência de gênero em mulheres estudantes universitárias: evidências sobre a prevalência e sobre os fatores associados
}

\author{
Taís Tasqueto Tassinari; Fernanda Honnef; Jaqueline Arboit; Tassiane Ferreira Langendorf; \\ Cristiane Cardoso de Paula; Stela Maris de Mello Padoin
}

Cómo citar este artículo:

Tasqueto, T., Honnef, F., Arboit, J., Ferreira, T., Cardoso, C., \& de Mello, S. M. (2022). Violência de gênero em mulheres estudantes universitárias: evidências sobre a prevalência e sobre os fatores associados. Acta Colombiana de Psicología, 25(1), 105-120. https://www.doi.org/10.14718/ACP.2022.25.1.8

Recibido, abril 7/2020; Concepto de evaluación, junio 28/2021; Aceptado, septiembre 17/2021

Taís Tasqueto Tassinari

ORCID: https://orcid.org/0000-0001-9878-0938

Universidade Federal de Santa Maria, Santa Maria, RS, Brasil

Fernanda Honnef

ORCID: http://orcid.org/0000-0002-1866-1611

Universidade Federal de Santa Maria, Santa Maria, RS, Brasil

Jaqueline Arboit

ORCID: http://orcid org/0000-0002-6610-5900

Universidade Federal de Santa Maria, Santa Maria, RS, Brasil

Tassiane Ferreira Langendorf

ORCID: https://orcid.org/0000-0002-5902-7449

Universidade Federal de Santa Maria, Santa Maria, RS, Brasil

Cristiane Cardoso de Paula

ORCID: http://orcid.org/0000-0003-4122-5161

Universidade Federal de Santa Maria, Santa Maria, RS, Brasil

Stela Maris de Mello Padoin

ORCID: http://orcid.org/0000-0003-3272-054X

Universidade Federal de Santa Maria, Santa Maria, RS, Brasil

Resumo

\begin{abstract}
A violência de gênero contra as mulheres está relacionada à desigualdade de gênero. Ela é compreendida como a ruptura de qualquer forma de integridade da mulher - física, sexual, psicológica, patrimonial, econômica ou moral - e pode ocorrer tanto no âmbito privado-familiar como nos espaços de trabalho e públicos. A violência de gênero repercute na saúde e na qualidade de vida das mulheres em qualquer âmbito social. No caso deste estudo, ela foi analisada no ambiente universitário e no desenvolvimento acadêmico delas. Nesse sentido, o presente estudo objetivou analisar as evidências acerca da prevalência da violência de gênero em mulheres estudantes universitárias e seus fatores associados. Trata-se de uma revisão integrativa da literatura realizada nas bases de dados LILACS, PubMed, CINAHL, ERIC e ASSIA, em que 22 artigos atenderam aos critérios de seleção. As evidências disponíveis na literatura indicaram diferentes formas de violência de gênero em mulheres universitárias, perpetradas por parceiros íntimos e outros agressores. A prevalência de violência de gênero em mulheres estudantes universitárias apontada nos estudos variou entre $1,3 \%$ e $85 \%$, diferindo de acordo com o tipo de violência. A violência está associada com fatores sociais e comportamentais, uma vez que as estudantes mais jovens apresentaram maiores índices de violência, assim como as de raça/etnia não branca, as que se identificavam como homossexuais ou bissexuais e as que tinham histórico de vitimização anterior. Evidências sobre o uso de álcool e outras drogas por parte das mulheres e a participação em irmandades sociais diferiram de acordo com o tipo de violência. Assim, conclui-se que a prevalência da violência de gênero na população de mulheres estudantes universitárias varia de acordo com a sua tipificação, é majoritariamente perpetrada por homens próximos a elas e está associada com marcadores sociais.

Palavras-chave: revisão, violência contra a mulher, estudantes, iniquidade de gênero, saúde da mulher.
\end{abstract}

1 Autor de correspondência: Stela Maris de Mello Padoin

Universidade Federal de Santa Maria, Centro de Ciências da Saúde, prédio 26, sala 1336. Avenida Roraima, 1000, bairro Camobi, Santa Maria, RS, Brasil. CEP: 97105-900. Telefone: (55 55)999713143 E-mail: stela.padoin@ufsm.br 


\title{
Violencia de género entre estudiantes universitarias: evidencia sobre prevalencia y factores asociados
}

\begin{abstract}
Resumen
La violencia de género contra las mujeres está relacionada con la desigualdad de género y se entiende que es la interrupción de cualquier forma de integridad de las mujeres. Puede ser del tipo físico, sexual, psicológico, patrimonial, económica o moral y puede ocurrir tanto en el entorno privado-familiar, como en el trabajo y los espacios públicos. La violencia de género tiene un impacto en la salud y la calidad de vida de las mujeres y puede generalizarse en entornos universitarios, lo que también implica el desarrollo académico de las mujeres. El presente estudio tuvo como objetivo analizar la evidencia sobre la prevalencia de la violencia de género en estudiantes universitarias y sus factores asociados. Esta es una revisión integral de la literatura realizada en las bases de datos LILACS, PubMed, CINAHL, ERIC y ASSIA, en la que 22 artículos cumplieron con los criterios de selección. La evidencia disponible en la literatura ha indicado diferentes formas de violencia de género en mujeres universitarias perpetradas por parejas íntimas y otros agresores. La prevalencia de la violencia de género en las estudiantes universitarias informadas en los estudios varió entre el 1,3\% y el 85\%, difiriendo según el tipo de violencia. La violencia está asociada a factores sociales y de comportamiento, ya que las estudiantes más jóvenes tuvieron tasas más altas de violencia, así como aquellos de raza/etnia no blanca, aquellos que se identificaron como homosexuales o bisexuales y los que tenían un historial de victimización Anterior. Las pruebas sobre el uso de alcohol y otras drogas por parte de las mujeres y la participación en hermandades sociales diferían según el tipo de violencia. Por lo tanto, se concluye que la prevalencia de la violencia de género en la población de estudiantes universitarias varía según su tipificación, en su mayoría es perpetrada por hombres cercanos a ellas y está asociada con marcadores sociales.

Palabras clave: revisión, violencia contra las mujeres, estudiantes, iniquidad de género, salud de la mujer.
\end{abstract}

\section{Gender-based violence among female university students: Evidence of prevalence and associated factors}

\begin{abstract}
Gender-based violence against women is related to gender inequality and is understood as the disruption of any form of the woman's integrity. It can be physical, sexual, psychological, patrimonial, economic or moral, and can occur in the private-family environment as well as in the work and public spaces. Gender-based violence affects the health and quality of life of women and can be disseminated in several ways in the university environments, which also affects the women's academic development. The present study aimed to analyze the evidence about the prevalence of gender-based violence among female university students and its associated factors. This is an integrative literature review performed in the LILACS, PubMed, CINAHL, ERIC and ASSIA databases, in which 22 articles met the selection criteria. Evidence available in the literature have indicated different forms of gender-based violence among college women perpetrated by intimate partners and other aggressors. The prevalence of gender-based violence in female university students reported in the studies varied between $1.3 \%$ and $85 \%$, differing according to the type of violence. Such violence is associated to social and behavioral factors, as younger students presented higher rates of violence, as well as those of a non-white race/ethnicity, those who identified themselves as homosexual or bisexual, and those with a previous history of victimization. Evidence about the use of alcohol and other drugs by women and their participation in social fraternities or sororities differed according to the type of violence. Thus, one may conclude that the prevalence of gender-based violence among the population of female university students varies according to its typification, is mostly perpetrated by men close to the women and is associated with social markers.
\end{abstract}

Keywords: review, violence against women, students, gender inequality, women's health 


\section{Introdução}

A violência de gênero compreende a violência contra as mulheres como um fenômeno relacionado à desigualdade de gênero, ou seja, a ruptura de qualquer forma de integridade da mulher (Saffioti, 2015). Essa violência pode ser física, sexual, psicológica, patrimonial, econômica ou assédio moral e ocorrer tanto no âmbito privado-familiar como nos espaços de trabalho e públicos (Bandeira, 2014; Hirigoyen, 2003; López, 2017). Ademais, a violência de gênero recai predominantemente sobre as mulheres, haja vista a construção histórica dos corpos femininos e o fenômeno do patriarcado, com relações assimétricas e de poder entre homens e mulheres baseadas no gênero (Bandeira, 2014; Scott, 1995).

As construções sociais de gênero moldam as responsabilidades destinadas a homens e mulheres, as quais afetam ou coíbem outras esferas da vida, como trabalho e educação, visto que o espaço público ainda é tido como masculino, enquanto cabe às mulheres o espaço privado (Biroli, 2018). Desse modo, a categoria gênero torna-se um determinante de acesso aos espaços de poder, em que as mulheres têm menores chances de participação em espaços públicos.

Contudo, os movimentos feministas e de mulheres foram, e são, responsáveis por promover o debate para modificar essas configurações sociais. As mulheres, além de modificarem sua própria condição na sociedade - a partir do ingresso delas no ensino superior e no mercado de trabalho, principalmente em países ocidentais (Ricoldi \& Artes, 2016) - , conquistaram direitos, o que provocou mudanças estruturais em geral (Santos et al., 2015). Uma pesquisadora de Ohio, nos Estados Unidos, apresenta que, a partir de 1990, constatou-se um predomínio nas matrículas de mulheres no ensino superior, sendo um fenômeno constatado no âmbito mundial (McDaniel, 2018). No Brasil, as mulheres ocupam a maioria das matrículas de graduação e pós-graduação $(57,2 \%)$, sendo também maioria entre os ingressantes $(55,2 \%)$ e concluintes $(61,4 \%)$ das instituições de ensino superior. Com relação aos 20 maiores cursos em número de matrículas, as mulheres são representação majoritária em 13 (Brasil, 2019).

Entretanto, além das situações de desqualificação, sexismo e outras formas de discriminação baseada no gênero, às quais as mulheres ainda estão sujeitas na sociedade (Navarro-Mastas \& Velásquez, 2016), no contexto das universidades, ocorrem diversas manifestações de violência, especialmente direcionadas às mulheres estudantes. Entre as formas que se expressam, podem-se citar os trotes agressivos, as práticas de racismo e homofobia entre estudantes, assédio sexual e moral, agressões físicas e humilhações sexistas. São recorrentes também os atos de violência sexual, na maioria das vezes, percebidos de forma naturalizada (Bandeira, 2017).

As repercussões da violência de gênero para a saúde e a qualidade de vida das mulheres são diversas. $O$ cotidiano delas é permeado por sofrimento, medo, lesões e marcas no corpo, dor, doenças e diversos danos físicos e psicológicos. Das lesões físicas mais comuns decorrentes de situações de violência, podem-se citar dores, hematomas e escoriações. Algumas mulheres podem apresentar lesões mais graves, como fraturas de ossos, queimaduras, entorses ou feridas com facas. As lesões podem ocorrer em qualquer parte do corpo, porém os mais comuns são os membros superiores e o rosto. Também são comuns agressões físicas na forma de empurrões, agarrar com força e socos (Domíngues Fernández et al., 2017; Organización Panamericana de la Salud \& Centers for Disease Control and Prevention, 2014).

Além disso, são mencionados a depressão (Barchi et al., 2018; Barros et al., 2016; Damra \& Abujilban, 2018; Kotan et al., 2020), ansiedade, baixa autoestima, abuso de drogas (Kachaeva \& Shport, 2017) e risco de suicídio (Barchi et al., 2018; Kavak et al., 2018). Desse modo, a saúde psicossocial das mulheres que sofrem violência está ameaçada (Tetikcok et al., 2016).

São identificados também problemas sociais, como isolamento, falta de apoio social e conflitos familiares (Leite et al., 2017; Souza Santos \& Jaeger, 2018). Além disso, o desenvolvimento humano das mulheres é comprometido, pois elas sentem-se oprimidas e apresentam dificuldades para a interação social, o que afeta seu relacionamento com amigos e familiares (Brock Carneiro et al., 2017).

Dessa maneira, as repercussões da violência de gênero interferem no desenvolvimento acadêmico e na rede social das mulheres no ambiente universitário (Pinto et al., 2016). Assim, essa evidência configura-se como uma barreira para suas aspirações educacionais, além do comprometimento de sua saúde e de seu bem-estar. 
Diante disso, o conhecimento relacionado à violência de gênero se faz necessário em diversos setores da sociedade, interessando diretamente para o seu enfrentamento as políticas sociais. Espera-se que as evidências disponíveis na literatura possam contribuir para subsidiar estratégias de prevenção à violência de gênero, bem como direcionar a atenção às especificidades das mulheres estudantes que vivenciam essa situação.

Assim, este estudo de revisão tem como objetivo analisar as evidências acerca da prevalência da violência de gênero em mulheres estudantes universitárias e seus fatores associados.

\section{Método}

Trata-se de um estudo de revisão integrativa, em que a síntese do conhecimento foi realizada por meio da análise, da sistematização e da integração de evidências de investigações publicadas, e apresenta potencial para contribuir para a indicação de tendências e avanços no conhecimento teórico. Este estudo de revisão foi guiado pelo método proposto por Whittemore e Knafl (2005), cujo primeiro passo consiste em determinar o problema de estudo e o objetivo da revisão.

Diante das considerações explanadas acima, a questão de revisão foi "Qual a prevalência da violência de gênero em mulheres estudantes universitárias e seus fatores associados?" A pergunta foi estruturada considerando os elementos do acrônimo CoCoPop (Aromataris \& Munn, 2020), em que "Co" é a condição estudada: violência de gênero; "Co", o contexto: universidade/ensino universitário; "Pop", a população: mulheres estudantes. A busca de artigos ocorreu de junho a agosto de 2018, nas bases de dados Literatura Latino Americana e do Caribe em Ciências da Saúde (LILACS), por meio do Portal Regional da Biblioteca Virtual em Saúde (BVS), PubMed, Cumulative Index to Nursing and Allied Health Literature (CINAHL), Education Resources Information Center (ERIC) e Applied Social Sciences Index and Abstracts (ASSIA).

Para a busca nas bases de dados, foram utilizados descritores controlados do Medical Subject Headings (MeSH), Descritores em Ciências da Saúde (DeCS) e descritores não controlados ou palavras-chave, os quais foram combinados com os operadores booleanos AND e OR. As combinações foram adaptadas de acordo com a especificidade de cada base de dados, a fim de propiciar uma busca ampla (Tabela 1).

Os critérios de inclusão dos estudos primários foram pesquisa transversal ou coorte, com descrição da prevalência de violência de quaisquer tipos (física, moral, psicológica e outras) contra a mulher e população do estudo composta apenas por mulheres estudantes de graduação ou pós-graduação; artigos publicados em inglês, português ou espanhol, de 2008 a 2018.

Foram excluídos os estudos realizados com a população de mulheres estudantes universitárias que analisavam a violência de gênero ao longo da vida, o abuso infantil e a violência na adolescência, e os estudos em que não foi possível definir a prevalência de violência ocorrida no período universitário.

A seleção dos estudos foi realizada por duas revisoras, de modo independente. Quando houve discordância, uma terceira revisora com expertise no tema avaliou a inclusão do estudo. Para operacionalizar a seleção dos estudos, utilizou-se o software gerenciador de referências bibliográficas Mendeley ${ }^{\circledR}$ (Yamakawa et al., 2014). Para descrever o percurso de seleção dos estudos, apresenta-se o fluxograma abaixo (Figura 1).

A extração dos dados dos estudos selecionados realizou-se mediante instrumento criado pelas revisoras, o qual abarcava a identificação do artigo, as referências, o objetivo, o resumo com palavras-chave, local e ano do estudo, as características metodológicas (tipo de estudo e técnica de coleta de dados), a amostra do estudo, a prevalência e o tipo de violência, bem como os fatores associados à violência.

A avaliação crítica dos estudos primários foi realizada considerando os níveis de evidência, de acordo com a classificação direcionada à questão clínica do estudo primário para o prognóstico, a predição ou a etiologia (Melnyk \& Fineout-Overholt, 2005). Nessa classificação, os níveis de evidência variam de 1 a 5 , segundo o tipo de estudo: nível 1 - síntese de estudos de coorte ou caso controle; nível 2 - único estudo de coorte ou caso controle; nível 3 - metassíntese de estudos qualitativos ou descritivos; nível 4 - um único estudo descritivo ou qualitativo; nível 5 - opinião de especialistas.

A análise dos dados foi realizada compreendendo a síntese dos estudos primários, bem como as comparações 
Tabela 1

Estratégias de busca realizadas nas bases de dados LILACS, PubMed, CINAHL, ERIC E ASSIA, 2019

\begin{tabular}{|c|c|}
\hline Base & Estratégia de busca \\
\hline LILACS & 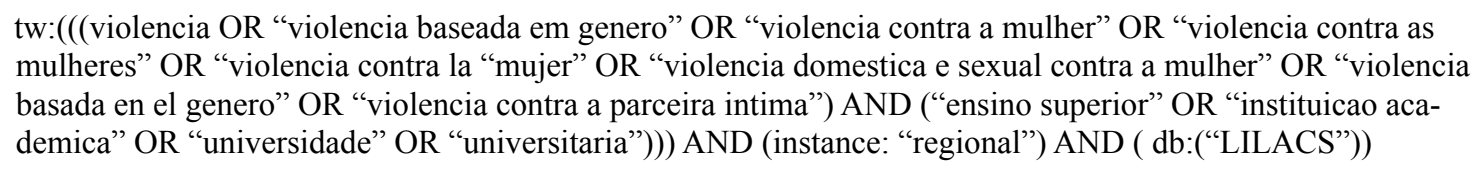 \\
\hline PubMed & 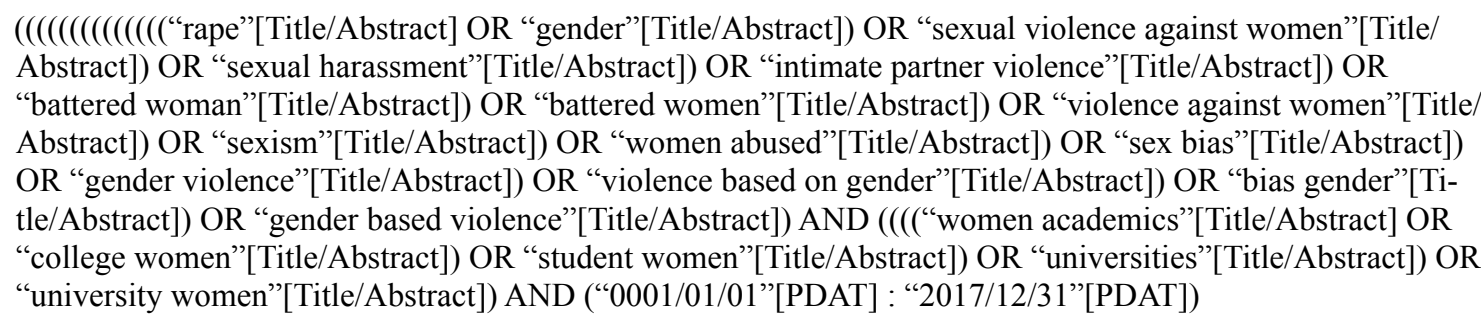 \\
\hline CINAHL & 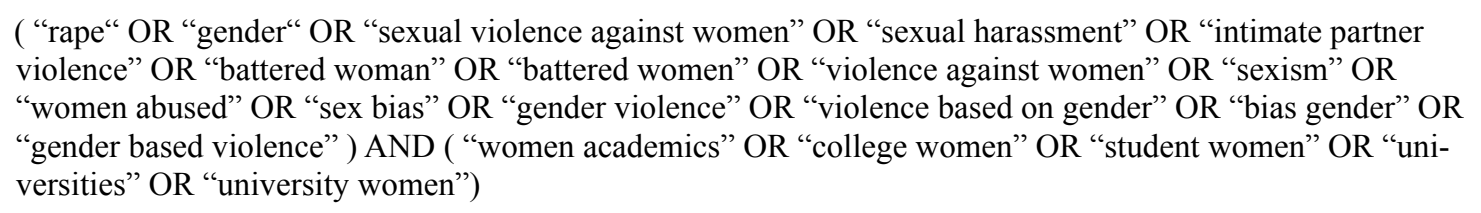 \\
\hline ERIC & $\begin{array}{l}\text { ("violence against women" OR "gender violence" OR "violence based on gender" OR "bias gender" OR "gen- } \\
\text { der based violence") AND ("women academics" OR "college women" OR "student women" OR universities } \\
\text { OR “university women") }\end{array}$ \\
\hline ASSIA & 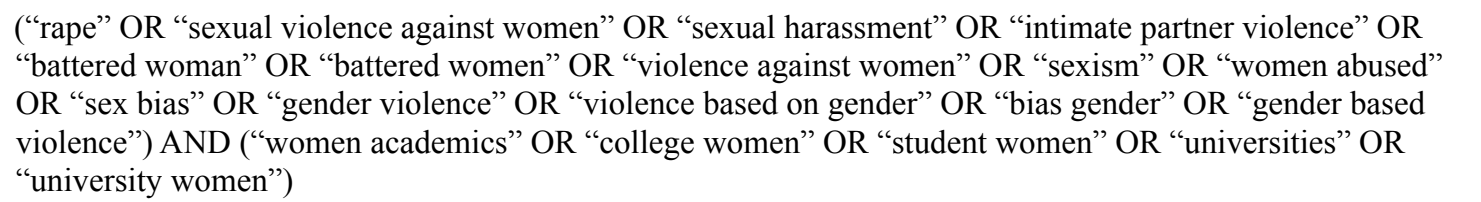 \\
\hline
\end{tabular}

Fonte: elaboração própria.

entre os principais resultados que respondem à pergunta de revisão, destacando diferenças e semelhanças entre os estudos. Além disso, realizou-se discussão com a categoria analítica de gênero, agregada à síntese das evidências, por meio de autoras que discorrem sobre esse tema.

\section{Resultados}

Para fins de caracterização, dos 22 estudos primários selecionados, $21(95,5 \%)$ foram publicados em inglês e um (4,5\%) em espanhol. Dezenove estudos originaram-se dos Estados Unidos (86,5\%). Os demais foram provenientes do Chipre (4,5\%), do México (4,5\%) e da Etiópia (4,5\%), com uma produção cada. Quanto ao ano de publicação, quatro artigos (18,2\%) foram publicados em 2009; três $(13,6 \%)$ em 2010; sete $(31,9 \%)$ em 2013 ; um $(4,5 \%)$ em 2014; cinco (22,8\%) em 2015 e dois (9\%) em 2016. Com relação ao delineamento dos estudos, 20 eram quantitativos transversais (91\%), um coorte $(4,5 \%)$ e um misto $(4,5 \%)$. A classificação do nível de evidência $(\mathrm{N})$ foi de $\mathrm{N} 4$ para $95,5 \%$ e N2 para 4,5\%. Identificou-se que os autores dos artigos pertencem às áreas de Ciências da Saúde e Ciências Sociais e Humanas.

As evidências disponíveis na literatura mencionam diferentes formas de violência de gênero em mulheres 
Figura 1

Fluxograma do processo de seleção dos estudos primários adaptado do Preferred Reporting Items for Systematic Review and Meta-Analyses (Prisma)

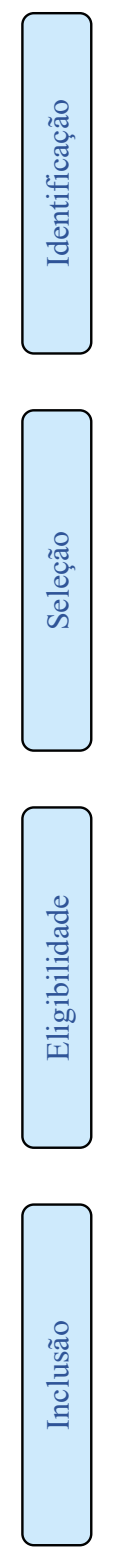

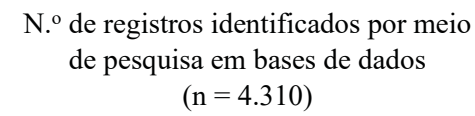

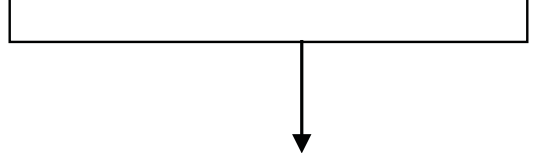

N. ${ }^{\circ}$ de registros após a remoção de duplicatas $(n=4.164)$
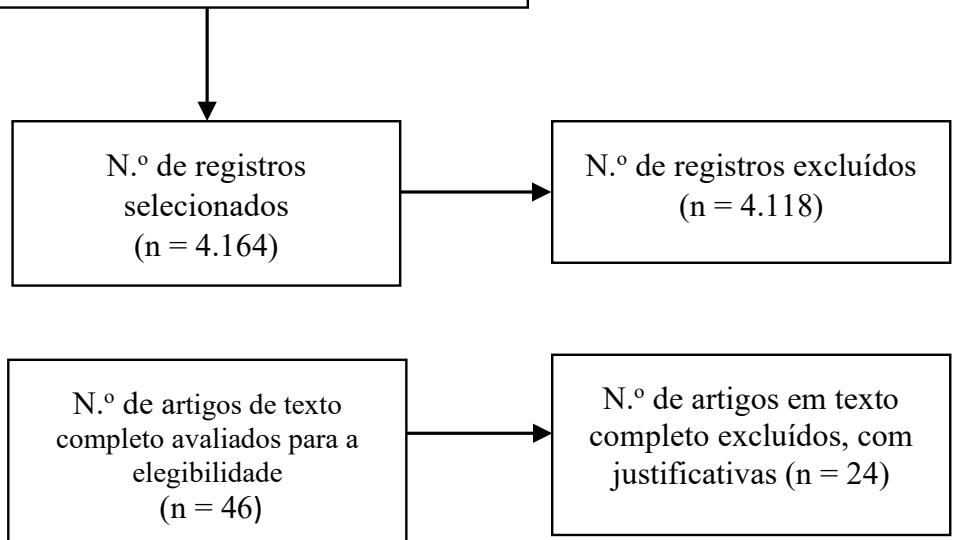

N. ${ }^{\circ}$ de estudos incluídos $(n=22)$
N. ${ }^{o}$ de registros adicionais identificados por outras fontes $(\mathrm{n}=0)$ $(\mathrm{n}=4.118)$ N. ${ }^{\circ}$ de artigos em texto justificativas $(\mathrm{n}=24)$

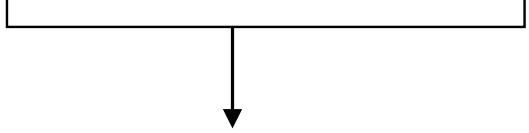

Fonte: adaptado do Prisma (2009).

universitárias, perpetradas por parceiros íntimos ou outros agressores (violência física, sexual, psicológica e moral). Os estudos apresentaram variações das amostras entre 73 e 10.541 estudantes, no contexto de universidades públicas e privadas. Alguns estudos tiveram como participantes estudantes em geral, e outros, apenas estudantes de cursos específicos. A prevalência de violência de gênero em mulheres estudantes universitárias apontada nos estudos variou entre 1,3\% (Kouta et al., 2013) e 85\% (Buelna et al., 2009), diferindo de acordo com o tipo de violência. Na Tabela 2, apresenta-se a síntese dos estudos primários. 
Tabela 2

Síntese dos estudos primários com a descrição de autores, ano de estudo, tipo de estudo, amostra e principais resultados, 2019

\begin{tabular}{|c|c|c|c|}
\hline Autor (ano) & Tipo de estudo & Amostra & Principais resultados \\
\hline $\begin{array}{l}\text { Amacker e Little- } \\
\text { ton (2013) }\end{array}$ & Transversal & $\begin{array}{l}\mathrm{N}=167 \\
\mathrm{NE}=\mathrm{G}\end{array}$ & $\begin{array}{l}\text { Cerca de } 20 \%(\mathrm{n}=33) \text { relataram ter sido vítimas de agressão sexual e quase } \\
23 \%(\mathrm{n}=38) \text { relataram ter sido vítimas de tentativa de agressão sexual. Ao } \\
\text { todo, } 31,1 \%(\mathrm{n}=52) \text { das participantes relataram pelo menos uma experiência } \\
\text { de vitimização sexual (tentativa, completada ou ambas). }\end{array}$ \\
\hline $\begin{array}{l}\text { Barrick et al. } \\
(2013)\end{array}$ & Transversal & $\begin{array}{l}\mathrm{N}=3951 \\
\mathrm{NE}=\mathrm{G}\end{array}$ & $\begin{array}{l}\text { Quase dois terços das mulheres relataram ter sofrido alguma violência por } \\
\text { parceiro íntimo pelo menos uma vez no ano anterior à pesquisa }(64,7 \%) \text {. Uma } \\
\text { porcentagem maior de mulheres apresentou violência verbal ou controladora } \\
\text { por parceiro íntimo }(63,7 \%) \text { do que violência física }(17,8 \%) \text { ou sexual }(1,4 \%) \text {. } \\
\text { Os resultados sugerem que formas de violência relativamente menos graves } \\
\text { eram as mais comuns. }\end{array}$ \\
\hline $\begin{array}{l}\text { Buelna et al. } \\
(2009)\end{array}$ & Transversal & $\begin{array}{l}\mathrm{N}=290 \\
\mathrm{NE}=\mathrm{G}\end{array}$ & $\begin{array}{l}\text { Dos itens de vitimização, } 79 \%(\mathrm{n}=228) \text { das pesquisadas referiram vitimi- } \\
\text { zação psicológica, } 37 \%(\mathrm{n}=107) \text { relataram vitimização física, } 44 \%(\mathrm{n}=128) \\
\text { relataram vitimização sexual e } 12 \%(\mathrm{n}=35) \text { relataram terem se ferido em } \\
\text { decorrência de vitimização. No total, } 85 \%(\mathrm{n}=247) \text { das mulheres relataram } \\
\text { pelo menos uma forma de vitimização. }\end{array}$ \\
\hline Buhi et al. (2009) & Transversal & $\begin{array}{l}\mathrm{N}=391 \\
\mathrm{NE}=\mathrm{G} \text { e } \\
\quad \mathrm{PG}\end{array}$ & $\begin{array}{l}\text { Um quinto }(\mathrm{n}=78) \text { das mulheres relataram vitimização de stalking enquanto } \\
\text { matriculadas em sua instituição atual. Entre as mulheres que relataram vitimi- } \\
\text { zação por stalking, os comportamentos de stalking mais frequentes relatados } \\
\text { foram ser observado de longe }(64,9 \% ; \mathrm{n}=51) \text {, ser seguido ou espiado }(62,8 \% \text {; } \\
\mathrm{n}=49) \text {, ser aguardado em locais externos ou internos, por exemplo, casa, aulas } \\
\text { ou trabalho }(53,2 \% ; \mathrm{n}=41) \text {, receber telefonemas não solicitados }(51,3 \% ; \mathrm{n}= \\
40) \text { e receber e-mails não solicitados }(44,9 \% ; \mathrm{n}=35) \text {. }\end{array}$ \\
\hline $\begin{array}{l}\text { Carey et al. } \\
(2015)\end{array}$ & Transversal & $\begin{array}{l}N=483 \\
N E=G\end{array}$ & $\begin{array}{l}\text { Durante o primeiro ano de faculdade, } 9 \%(n=43) \text { relataram estupro forçado } \\
\text { tentado ou concluído e } 15,4 \%(n=74) \text { relataram estupro incapacitado tentado } \\
\text { ou concluído. }\end{array}$ \\
\hline $\begin{array}{l}\text { Dixon et al. } \\
(2015)\end{array}$ & Transversal & $\begin{array}{l}\mathrm{N}=887 \\
\mathrm{NE}=\mathrm{G}\end{array}$ & $\begin{array}{l}69,2 \% \text { das participantes }(\mathrm{n}=647) \text { relataram estar em relacionamentos não vio- } \\
\text { lentos e } 30,8 \%(\mathrm{n}=240) \text { das participantes relataram alguma forma de violência } \\
\text { por parceiro íntimo do tipo física em seus relacionamentos. O padrão mais co- } \\
\text { mum de violência por parceiro íntimo relatado foi perpetração apenas }(11,9 \% \text {; } \\
\mathrm{n}=111) \text {, seguido por bidirecional, em que ambos perpetraram a violência } \\
(10,6 \% ; \mathrm{n}=99) \text {, e vitimização apenas }(7,4 \% ; \mathrm{n}=69) \text {. }\end{array}$ \\
\hline $\begin{array}{l}\text { Fontenot e Fanta- } \\
\text { sia }(2010)\end{array}$ & Transversal & $\mathrm{N}=1.401$ & $\begin{array}{l}10,4 \%(\mathrm{n}=146) \text { das mulheres relataram que já tiveram relações sexuais com } \\
\text { alguém mesmo depois de ter dito ou mostrado que não queria; } 3,4 \%(\mathrm{n}=47) \\
\text { relataram incerteza sobre ter relações sexuais com alguém após ter dito ou } \\
\text { mostrado que não queria. Das que indicaram ter sofrido sexo indesejado, } 37 \% \\
(\mathrm{n}=54) \text { indicaram que ocorreu nos últimos } 12 \text { meses. }\end{array}$ \\
\hline $\begin{array}{l}\text { Hossain et al. } \\
(2014)\end{array}$ & Transversal & $\begin{array}{c}\mathrm{N}=10.541 \\
\mathrm{NE}=\mathrm{G}\end{array}$ & $\begin{array}{l}15,6 \%(\mathrm{n}=1.644) \text { da amostra de estudantes universitárias relatou ter sofrido } \\
\text { violência sexual e } 84,4 \%(\mathrm{n}=8.897) \text { relataram não ter sofrido violência sexual. }\end{array}$ \\
\hline $\begin{array}{l}\text { Katz e Rich } \\
(2015)\end{array}$ & Coorte & $\begin{array}{l}\mathrm{N}=99 \\
\mathrm{NE}=\mathrm{G}\end{array}$ & $\begin{array}{l}\text { Cerca de } 35 \%(\mathrm{n}=34) \text { da amostra relataram pelo menos um incidente de } \\
\text { violência por parceiro íntimo física, e cerca de } 57 \%(\mathrm{n}=56) \text { relataram pelo } \\
\text { menos um incidente de violência por parceiro íntimo sexual antes de seu rompi- } \\
\text { mento mais recente. Em relação à covitimização pré-rompimento (durante o } \\
\text { namoro }), 34 \%(\mathrm{n}=33) \text { relataram ausência de violência por parceiro íntimo, } \\
30,3 \%(\mathrm{n}=30) \text { relataram violência por parceiro íntimo sexual, } 9,1 \%(\mathrm{n}=9) \\
\text { relataram violência por parceiro íntimo física e } 26,3 \%(\mathrm{n}=26) \text { relataram tanto } \\
\text { violência por parceiro íntimo física e sexual. Após o rompimento, cerca de } \\
19,2 \%(\mathrm{n}=19) \text { das mulheres relataram pelo menos um incidente de violência } \\
\text { por parceiro íntimo física e } 29,3 \%(\mathrm{n}=29) \text { relataram pelo menos um incidente } \\
\text { de violência por parceiro íntimo sexual. }\end{array}$ \\
\hline
\end{tabular}




\begin{tabular}{|c|c|c|c|}
\hline Autor (ano) & Tipo de estudo & Amostra & Principais resultados \\
\hline $\begin{array}{l}\text { Kouta et al. } \\
(2013)\end{array}$ & Misto & $\begin{array}{l}\mathrm{N}=476 \\
\mathrm{NE}=\mathrm{G}\end{array}$ & $\begin{array}{l}\text { A porcentagem de mulheres jovens que têm uma experiência sexual indesejada } \\
\text { (como sexo oral, tocando genitais sob a roupa) é de } 12 \% \text {. Tentativas de estupro } \\
\text { foram relatadas por } 1,3 \%(n=5) \text { das participantes, } 1,9 \%(n=7) \text { relataram } \\
\text { ter sido forçadas a fazer sexo oral e } 1,9 \%(n=7) \text { relataram que haviam sido } \\
\text { estupradas }\end{array}$ \\
\hline $\begin{array}{l}\text { Krebs et al. } \\
(2009)\end{array}$ & Transversal & $\begin{array}{c}\mathrm{N}=5.446 \\
\mathrm{NE}=\mathrm{G}\end{array}$ & $\begin{array}{l}19 \%(\mathrm{n}=1073) \text { das mulheres relataram ter sofrido agressão sexual completa ou } \\
\text { tentativa de agressão sexual desde que ingressaram na faculdade. Desde que in- } \\
\text { gressou na faculdade, } 13,7 \%(\mathrm{n}=782) \text { das mulheres experimentaram agressão } \\
\text { sexual completa e } 12,6 \%(\mathrm{n}=682) \text { das mulheres experimentaram tentativa } \\
\text { de agressão sexual. Aproximadamente } 11 \%(\mathrm{n}=651) \text { das mulheres sofreram } \\
\text { agressão sexual enquanto estavam incapacitadas desde que ingressaram na } \\
\text { faculdade. A agressão sexual ativada por álcool ou outras drogas foi vivenciada } \\
\text { por } 7,8 \%(\mathrm{n}=466) \text { das mulheres desde que ingressaram na faculdade. Durante } \\
\text { a faculdade, a prevalência de agressão sexual incapacitada concluída é consid- } \\
\text { eravelmente maior do que no período anterior à faculdade. }\end{array}$ \\
\hline $\begin{array}{l}\text { Lindquist et al. } \\
\text { (2013) }\end{array}$ & Transversal & $\begin{array}{c}\mathrm{N}=3.951 \\
\mathrm{NE}=\mathrm{G}\end{array}$ & $\begin{array}{l}4,5 \% \text { das mulheres sofreram agressões sexuais fisicamente forçadas desde a } \\
\text { entrada na faculdade }(n=188) \text { e } 6,2 \% \text { das mulheres sofreram agressão sexual } \\
\text { incapacitada desde que ingressaram na faculdade }(n=250) \text {. }\end{array}$ \\
\hline $\begin{array}{l}\text { Messman-Moore } \\
\text { et al. (2013) }\end{array}$ & Transversal & $\begin{array}{l}\mathrm{N}=353 \\
\mathrm{NE}=\mathrm{G}\end{array}$ & $\begin{array}{l}\text { Aproximadamente } 15,6 \%(\mathrm{n}=55) \text { da amostra relataram estupro envolvido com } \\
\text { álcool, indicando que haviam vivenciado sexo oral, vaginal ou anal indesejado, } \\
\text { porque não conseguiram dar consentimento ou resistir ao agressor devido ao } \\
\text { seu próprio uso de álcool (ou substância). }\end{array}$ \\
\hline $\begin{array}{l}\text { Herrera Paredes } \\
\text { e Arena Ventura } \\
(2010)\end{array}$ & Transversal & $\begin{array}{c}\mathrm{N}=73 \\
\mathrm{NE}=\mathrm{G}\end{array}$ & $\begin{array}{l}\text { Em relação à presença de violência física, } 91,8 \%(n=67) \text { disseram nunca ter } \\
\text { sido maltratados pelo parceiro, no entanto, } 2,7 \%(n=2), 1,4 \%(n=1) \text { e } 4,1 \% \\
(n=3) \text { referiram que sempre, às vezes e raramente, respectivamente, vivencia- } \\
\text { ram comportamentos agressivos por seu parceiro. }\end{array}$ \\
\hline $\begin{array}{l}\text { Sandberg et al. } \\
\text { (2019) }\end{array}$ & Transversal & $\begin{array}{l}N=133 \\
N E=G\end{array}$ & $\begin{array}{l}21 \%(\mathrm{n}=27) \text { das participantes relataram sofrer vitimização por agressão física } \\
\text { em um relacionamento romântico durante o período de acompanhamento de } \\
\text { seis meses. Dezoito }(13,5 \%) \text { participantes relataram ter experimentado dois ou } \\
\text { mais tipos de vitimização por violência por parceiro íntimo durante o período } \\
\text { de acompanhamento. }\end{array}$ \\
\hline $\begin{array}{l}\text { Schry e White } \\
(2013)\end{array}$ & Transversal & $\begin{array}{l}\mathrm{N}=672 \\
\mathrm{NE}=\mathrm{G}\end{array}$ & $\begin{array}{l}25,7 \% \text { das participantes relataram contato sexual nos últimos } 12 \text { meses } \\
(\mathrm{n}=173) ; 14,1 \% \text { das participantes relataram tentativa de coerção nos últimos } \\
12 \text { meses }(\mathrm{n}=95) ; 8 \% \text { das estudantes relataram coerção nos últimos } 12 \text { meses } \\
(\mathrm{n}=54) ; 16,1 \% \text { das participantes relataram tentativa de estupro nos últimos } \\
12 \text { meses }(\mathrm{n}=108) \text { e } 14,9 \% \text { das participantes relataram estupro nos últimos } 12 \\
\text { meses }(\mathrm{n}=100) .\end{array}$ \\
\hline $\begin{array}{l}\text { Sutherland et al. } \\
\text { (2015) }\end{array}$ & Transversal & $\begin{array}{l}\mathrm{N}=972 \\
\mathrm{NE}=\mathrm{G} \\
\text { e PG }\end{array}$ & $\begin{array}{l}\text { A violência por parceiro íntimo foi relatada por } 20,6 \%(\mathrm{n}=201) \text { das entrevista- } \\
\text { das. Quase } 8 \% \text { das universitárias }(\mathrm{n}=76) \text { relataram um ato de coerção reprodu- } \\
\text { tiva (coerção na gravidez ou sabotagem no controle da natalidade). Quase } 82 \% \\
(\mathrm{n}=62 / 76) \text { das mulheres que relataram coerção reprodutiva foram instruídas a } \\
\text { não usar o controle da natalidade e } 26 \%(\mathrm{n}=20 / 76) \text { das mulheres foram força- } \\
\text { das a fazer sexo sem preservativo ou outro método de controle da natalidade. } \\
\text { Ser instruída a não usar nenhum controle de natalidade foi o ato mais comum } \\
\text { de coerção na gravidez relatado }(\mathrm{n}=62) \text {. Quase } 14 \%(\mathrm{n}=11 / 76) \text { das mulheres } \\
\text { que relataram coerção reprodutiva tiveram parceiros que tiraram preservativos } \\
\text { durante o sexo. }\end{array}$ \\
\hline $\begin{array}{l}\text { Sutherland et al. } \\
\text { (2016) }\end{array}$ & Transversal & $\begin{array}{l}\mathrm{N}=615 \\
\mathrm{NE}=\mathrm{G}\end{array}$ & $\begin{array}{l}\text { Nos últimos seis meses, aproximadamente } 8,2 \% \text { dos participantes }(\mathrm{n}=51) \\
\text { sofreram violência por parceiro íntimo/violência sexual ou ambos por um } \\
\text { parceiro ou ex-parceiro. Os atos de violência relatados foram atividades sexuais } \\
\text { indesejadas forçadas }(13,2 \% ; \mathrm{n}=29) \text {, violência física }(9,1 \% ; \mathrm{n}=20) \text { e sexo } \\
\text { forçado }(3,6 \% ; \mathrm{n}=8) \text {, e relataram medo do parceiro ou ex-parceiro }(3,6 \% \text {; } \\
\mathrm{n}=8) \text {. }\end{array}$ \\
\hline
\end{tabular}




\begin{tabular}{|c|c|c|c|}
\hline Autor (ano) & Tipo de estudo & Amostra & Principais resultados \\
\hline $\begin{array}{l}\text { Messman-Moore } \\
\text { et al. (2009) }\end{array}$ & Transversal & $\begin{array}{l}\mathrm{N}=276 \\
\mathrm{NE}=\mathrm{G}\end{array}$ & $\begin{array}{l}\text { Das mulheres que completaram as quatro sessões }(\mathrm{n}=276), 9 \%(\mathrm{n}=26) \\
\text { relataram estupro durante o período do estudo. A maioria das agressões que } \\
\text { ocorreram durante o estudo também foi relacionada à substância }(88 \%, \mathrm{n}=23) \text {; } \\
69 \%(\mathrm{n}=18) \text { foram devido à incapacidade da respondente de consentir ou re- } \\
\text { sistir devido ao uso de álcool ou substâncias na ausência de força, } 19 \%(\mathrm{n}=5) \\
\text { foram devido a uma combinação de comprometimento relacionado à substância } \\
\text { e à força ou ameaças de força, e } 12 \%(\mathrm{n}=3) \text { foram causados por ameaças ou } \\
\text { uso da força na ausência de comprometimento relacionado à substância. }\end{array}$ \\
\hline Tora (2013) & Transversal & $\begin{array}{l}\mathrm{N}=374 \\
\mathrm{NE}=\mathrm{G}\end{array}$ & $\begin{array}{l}\text { Entre as vítimas de tentativa de estupro, } 30,9 \%(\mathrm{n}=25) \text { vivenciaram-no durante } \\
\text { o ano de matrícula na universidade, enquanto } 11,1 \%(\mathrm{n}=9) \text { das entrevistadas } \\
\text { experimentaram isso no segundo ano da universidade ou acima. Dos casos de } \\
\text { estupro relatados, } 32,1 \%(\mathrm{n}=9) \text { foram vítimas durante o ano de matrícula na } \\
\text { universidade, e a experiência de estupro no segundo ano da universidade ou aci- } \\
\text { ma constituiu } 10,7 \%(\mathrm{n}=3) \text {. Entre as entrevistadas que relataram sofrer assédio } \\
\text { físico (violência sexual), } 31,1 \%(\mathrm{n}=23) \text { sofreram durante o ano de matrícula } \\
\text { na universidade e } 13,5 \%(\mathrm{n}=10) \text { relataram sofrer no segundo ano da universi- } \\
\text { dade ou acima. Entre as mulheres que relataram sofrer assédio verbal (violência } \\
\text { sexual), } 38,7 \%(\mathrm{n}=24) \text { sofreram no ano de matrícula da universidade e } 9,7 \% \\
(\mathrm{n}=6) \text {, no segundo ano na universidade ou acima. Das mulheres que indicaram } \\
\text { sofrer iniciação sexual forçada, } 41,2 \%(\mathrm{n}=14) \text { vivenciaram durante o ano de } \\
\text { matrícula, e } 8,8 \%(\mathrm{n}=3) \text { vivenciaram durante o segundo ano da universidade } \\
\text { ou acima. }\end{array}$ \\
\hline $\begin{array}{l}\text { Wigderson e Katz } \\
(2015)\end{array}$ & Transversal & $\begin{array}{l}\mathrm{N}=254 \\
\mathrm{NE}=\mathrm{G}\end{array}$ & $\begin{array}{l}\text { Cerca de } 15,7 \%(\mathrm{n}=40) \text { da amostra relataram uma ou mais experiências de } \\
\text { agressão sexual durante a faculdade. }\end{array}$ \\
\hline Yoon et al. (2010) & Transversal & $\begin{array}{l}\mathrm{N}=410 \\
\mathrm{NE}=\mathrm{G}\end{array}$ & $\begin{array}{l}\text { Mais de } 50 \% \text { de todas as mulheres }(\mathrm{n}=226) \text { relataram ter sido assediadas } \\
\text { sexualmente pelo menos uma ou duas vezes }(33 \% ; \mathrm{n}=135) \text {, enquanto uma por- } \\
\text { centagem significativa }(22,2 \% ; \mathrm{n}=91) \text { relatou ter sido vítima de assédio sexual } \\
\text { em várias ocasiões. }\end{array}$ \\
\hline
\end{tabular}

$\mathrm{N}$ = número de participantes/amostra; $\mathrm{NE}=$ nível de ensino; $\mathrm{G}=$ graduação; $\mathrm{PG}=$ pós-graduação.

Fonte: elaboração própria.

\section{Discussão}

As evidências encontradas nesta revisão demonstram que as mulheres estão sujeitas a diversas formas de violência durante o período em que estão matriculadas na universidade, as quais estão associadas a determinados fatores sociais e comportamentais. Dos quatro países dos quais os artigos se originaram, Estados Unidos, México e Etiópia apresentaram aumento das matrículas de mulheres em universidades entre 1990 e 2008 (McDaniel, 2018), o que pode indicar a representatividade desses países no corpus do estudo em tela.

Os estudos confirmam que a maior parte das violências sofridas pelas mulheres é perpetrada por homens, com destaque para os parceiros íntimos, namorados ou companheiros (Barrick et al., 2013; Buelna et al., 2009; Dixon et al., 2015; Herrera Paredes \& Arena Ventura, 2010; Sandberg et al.,
2019; Sutherland et al., 2015). Outros estudos demonstram que, além dos agressores já citados, as mulheres sofrem violência também dos ex-parceiros (Katz \& Rich, 2015; Sutherland et al., 2016), de amigos (Buhi et al., 2009; Kouta et al., 2013; Lindquist et al., 2013), colegas, conhecidos, colega de trabalho ou empregador, professor ou assistente de ensino, colega de quarto, membro da fraternidade ou alguma outra pessoa (Buhi et al., 2009; Lindquist et al., 2013; Tora, 2013). Percebe-se que as mulheres estudantes, muitas vezes, não estão seguras em seus círculos sociais e são agredidas por pessoas de sua confiança.

A violência de gênero perpetrada contra mulheres universitárias tem associação com a faixa etária, visto que mulheres mais jovens relatam índices mais altos de violência por parceiro íntimo (Barrick et al., 2013). Além disso, mulheres entre 18 e 19 anos tem 1,6 mais chances de sofrer violência sexual quando comparadas a mulheres 
mais velhas da mesma população (Hossain et al., 2014). Da mesma forma, mulheres com menos de 20 anos de idade têm maior probabilidade de sofrer tentativa de estupro (Tora, 2013). Entretanto, para a violência sexual, há divergência desse resultado, uma vez que, em um estudo, o grupo agredido sexualmente era significativamente mais velho do que o grupo que não sofreu esse tipo de agressão (Wigderson \& Katz, 2015).

As mulheres brancas foram menos propensas a sofrer qualquer violência por parceiro íntimo ( 0,87 vezes) (Barrick et al., 2013). Ser uma mulher negra foi significativamente associado com violência sexual (1,35 vezes mais chances) (Hossain et al., 2014), tentativa de estupro e assédio sexual (Yoon et al., 2010), e mulheres brancas eram mais propensas a serem assediadas uma ou duas vezes, enquanto as estudantes negras eram mais propensas a serem assediadas com frequência (Yoon et al., 2010). Também, com relação à coerção reprodutiva, um dos estudos evidenciou taxas mais altas entre as mulheres hispânicas (Sutherland et al., 2015). Percebe-se, assim, que algumas formas de violência de gênero se mostraram relacionadas à raça/etnia.

Esses dados refletem o racismo ainda presente não apenas na sociedade em geral, mas também dentro das instituições, neste caso, as universidades. Ainda, tais dados reforçam as diferenças na forma como as violências são direcionadas às mulheres, o que converge com o conceito de interseccionalidade, em que se reconhecem as consequências estruturais e dinâmicas da interação entre dois ou mais eixos de subordinação (Crenshaw, 2002).

Em um dos estudos, as mulheres que relataram ser atraídas apenas por mulheres tinham maior probabilidade de vivenciar violência por parceiro íntimo quando comparadas às que se sentiam atraídas apenas por homens. Assim, as mulheres homossexuais tinham 3,31 vezes mais chances de sofrer qualquer violência por parceiro íntimo, 1,98 vezes mais chances de sofrer violência física ou sexual por parceiro íntimo e 3,47 vezes mais chances de sofrer violência verbal ou controladora por parceiro íntimo. Ainda, as mulheres bissexuais tinham maior probabilidade ( 1,45 vezes) de sofrer violência por parceiro íntimo tanto física ou sexual quanto verbal ou controladora (Barrick et al., 2013).

Nesse sentido, cabe considerar a relação entre orientação-atração sexual e violência de gênero. Assim, destaca-se a reflexão de Bandeira (2014), que afirma que a violência de gênero ocorre motivada pelas expressões de desigualdade baseadas nas relações hierárquicas de gênero, porém salientando a necessidade de considerar as modificações advindas de algumas marcas como idade, classe, orientação sexual, dentre outras.

Além disso, a presença de crenças patriarcais, sexistas e tradicionais com relação aos papéis de gênero, apresenta relação causal no comportamento do agressor e da vítima. Por exemplo, em estudo desenvolvido no México, as mulheres justificam a agressão pelo namorado em situações de infidelidade (Orozco Vargas et al., 2021). Em outro estudo, desenvolvido no Peru, as ações punitivas de violência sexual envolvem uma combinação de ameaças e estão associados a múltiplos fatores como antecedentes de agressão física entre pai e mãe, questões econômicas e tolerância social à violência de gênero (Enríquez-Canto et al., 2020).

Quanto ao status de relacionamento das mulheres estudantes, este é apontado como um fator relacionado à violência de gênero, havendo divergências nas evidências identificadas. Mulheres casadas ou em parceria doméstica foram mais propensas a relatar qualquer tipo de violência por parceiro íntimo ( 2,80 vezes) e violência por parceiro íntimo do tipo verbal ou controladora ( 2,89 vezes). Também as estudantes que namoraram ou tiveram pelo menos um parceiro sexual desde que entraram na faculdade tiveram mais chances de relatar todos os tipos de violência por parceiro íntimo (Barrick et al., 2013). Isso corrobora com estudo desenvolvido com adolescentes brasileiros $(n=403)$, idade entre 14 e 19 anos $(M=16,73$ anos), que relataram ter perpetrado algum tipo de violência nas relações afetivo-sexuais na adolescência. Foi evidenciado que a violência que ocorreu com mais frequência foi a psicológica, verbal, emocional (92\%), seguida da violência sexual (37\%) e da violência física (27\%) (Borges et al., 2020).

Um estudo desenvolvido na Etiópia evidenciou que estudantes com experiência anterior de relação sexual tiveram mais chances $(0,099)$ de relatar tentativa de estupro e assédio físico $(0,429)$, em comparação com aquelas que não tinham experiência de relações sexuais (Tora, 2013). Corroborando com esses dados, investigação realizada nos Estados Unidos mostrou que apenas uma das 55 mulheres sexualmente abstinentes (2,5\%) relatou agressão sexual, enquanto quase um quinto das mulheres não abstinentes $(19,6 \%, n=39)$ relatou sofrer agressão; assim, a abstinência sexual parecia proteger as mulheres de agressão sexual (Wigderson \& Katz, 2015). 
Também o número de parceiros sexuais foi significativamente associado à experiência de sexo indesejado (Fontenot \& Fantasia, 2010) e violência sexual (Hossain et al., 2014). Mulheres que relataram ter dois ou mais parceiros sexuais foram 3,17 vezes mais propensas a sofrer violência sexual em comparação com aquelas que não tinham parceiro sexual (Hossain et al., 2014). Por sua vez, também há evidências que demonstram que mulheres estudantes que não tinham namorado tiveram maior probabilidade de vivenciar estupro e relataram maiores níveis de assédio físico (Tora, 2013).

O pertencimento a uma irmandade social (associação de estudantes com um interesse em comum, que pode contemplar a moradia e a organização de atividades conjuntas) foi outro aspecto que resultou em divergências nos resultados dos estudos. As mulheres que participavam de irmandades relataram ser mais perseguidas do que as que não pertenciam a esse tipo de organização (Buhi et al., 2009). Do mesmo modo, tornar-se membro de uma irmandade parecia estar significativamente associado à violência sexual. As alunas que relataram pertencer a um grupo social de fraternidade ou irmandade tiveram mais de 1,33 vezes mais chances de sofrer violência sexual em comparação com aquelas que não eram membros desses grupos sociais (Hossain et al., 2014). Entretanto, outro estudo evidenciou que ser membro de uma irmandade foi negativamente associado à experiência de violência física ou sexual por parceiro íntimo (Barrick et al., 2013).

Outro fator que tem relação com a violência de gênero entre mulheres estudantes universitárias é a situação de moradia. Estudos demonstraram que as mulheres que moravam no campus apresentaram níveis mais altos de perseguição (Buhi et al., 2009) e tiveram 1,6 vezes mais chance de sofrer violência sexual (Hossain et al., 2014). Com relação ao período de maior risco, as experiências de assédio verbal e iniciação sexual forçada apresentaram maior probabilidade de ocorrer no ano de ingresso na universidade (Tora, 2013).

O uso de álcool pelas mulheres estudantes também se mostra como um fator de risco para sofrer violência sexual (Hossain et al., 2014; Wigderson \& Katz, 2015), bem como o comportamento de compulsão alcoólica (Hossain et al., 2014) ou consumo excessivo (compreendido como mais de cinco doses seguidas) (Fontenot \& Fantasia, 2010). Contudo, as mulheres que relataram consumo excessivo de álcool pelo menos uma vez por mês desde que entraram na universidade tiveram menos probabilidade de vivenciar qualquer violência por parceiro íntimo (Barrick et al., 2013).

Ainda, em um dos estudos, o uso de maconha esteve associado significativamente à violência sexual (Hossain et al., 2014). Além disso, mulheres que usaram outras drogas ilícitas durante o período da faculdade tinham maior probabilidade de sofrer violência física ou sexual por parceiro íntimo do que as que não o fizeram. Entretanto, no mesmo estudo, o uso de maconha não esteve associado à violência por parceiro íntimo (Barrick et al., 2013).

As evidências acerca da revitimização foram diversas, $o$ que demonstra que dependem do tipo de violência ocorrida. Vivenciar agressão sexual fisicamente forçada antes de ingressar na faculdade foi associado a um risco aumentado (1,54 vezes) de sofrer qualquer violência por parceiro íntimo (Barrick et al., 2013) e aumentou as taxas de estupro forçado durante a faculdade (Carey et al., 2015). Ademais, agressão sexual incapacitada (aquela em que a mulher não é capaz de consentir) antes de ingressar na faculdade foi associada a um aumento (1,81 vezes) no risco de sofrer violência física ou sexual por parceiro íntimo (Barrick et al., 2013) e aumentou as taxas de estupro incapacitado e estupro forçado durante a faculdade (Carey et al., 2015).

Além disso, mulheres estudantes que foram vítimas de estupro ou de tentativa de estupro durante a faculdade também relataram sofrer mais perseguição. Essa vitimização consiste em situações como ser observado de longe, ser seguido ou espiado, ser aguardado em locais externos ou internos, como casa, aulas ou trabalho, receber telefonemas, e-mails ou presentes não solicitados, entre outras. O perseguidor mais frequente foi o namorado ou ex-namorado, seguido de colega de aula, conhecido e amigo, podendo ser também colega de trabalho, professor ou assistente de ensino (Buhi et al., 2009).

Com relação à revitimização, ter sofrido violência por parceiro íntimo previamente foi uma evidência associada a um aumento de risco de 3,68 vezes para a vitimização subsequente (Messman-Moore et al., 2009). Ademais, um histórico de coerção reprodutiva foi associado a relatos de violência por parceiro íntimo (Sutherland et al., 2015).

Um dos estudos analisados apresentou resultados quanto à violência por parceiro íntimo antes e depois do término do relacionamento. A violência física antes do rompimento foi 
um preditivo significativo para a violência física pós-ruptura, da mesma forma que a violência sexual cometida pelo parceiro antes do rompimento precedeu à violência sexual por parceiro íntimo após o rompimento (Katz \& Rich, 2015).

A partir desses dados, pode-se observar que as mulheres estudantes estão em risco de sofrer violência mesmo após o término do relacionamento com o agressor. Nesse sentido, Saffioti (2001) aponta que, para além da concepção territorial, existe a concepção simbólica de espaço privado, o que confere aos homens o direito de exercer seu poder sobre as mulheres mesmo após o rompimento da relação. Além disso, a autora acrescenta sobre a banalização da violência no espaço privado por parte do Estado, que não intervém para enfrentar esse problema, o que reforça a dificuldade das mulheres estudantes em cessar a violência sofrida.

\section{Conclusões}

O presente estudo permitiu identificar que a violência de gênero em mulheres estudantes universitárias varia de acordo com a sua tipificação e é majoritariamente perpetrada por homens que fazem parte de seu círculo social. Conclui-se também que as violências têm associação com marcadores sociais, uma vez que estudantes jovens, negras e não heterossexuais apresentam maior risco de sofrer violência de gênero no período em que se encontram na universidade. Além disso, outros fatores evidenciados associados à prevalência foram ter maior número de parceiros sexuais, morar no campus universitário, usar álcool e outras drogas e ter uma experiência prévia de violência. Ao analisar criticamente os artigos por meio da classificação de evidências dos estudos primários, a maioria (20) possuía nível de evidência 4 (estudos transversais), o que indica a necessidade de estudos primários do tipo coorte no tema em questão.

Recomenda-se ampliar e direcionar as estratégias de prevenção de violência em instituições universitárias. Tais estratégias podem ocorrer por meio de implementação de políticas, discussões e eventos sobre igualdade de gênero, bem como pesquisas para a identificação da prevalência da violência em ambientes universitários.

\section{Referências}

Amacker,A. M., \& Littleton, H. L. (2013). Perceptions of similarity and responsibility attributions to an acquaintance sexual assault victim. Violence Against Women, 19(11), 1384-1407. https://doi.org/10.1177/1077801213514860

Aromataris, E., \& Munn, Z. (eds.). (2020). JBI Manual for Evidence Synthesis. JBI. https://synthesismanual.jbi.global. https://doi.org/10.46658/JBIMES-20-01

Bandeira, L. M. (2014). Violência de gênero: a construção de um campo teórico e de investigação. Revista Sociedade e Estado, 29(2). https://doi.org/10.1590/ S0102-69922014000200008

Bandeira, L. M. (2017) Trotes, assédios e violência sexual nos campi Universitários no Brasil. GÊNERO, 17(2), 49-79. https://periodicos.uff.br/revistagenero/article/ view/31263/18352

Barchi, F., Winter, S., Dougherty, D., \& Ramaphane, P. (2018). Intimate partner violence against women in northwestern Botswana: The maun women's study. Violence Against Women, 24(16), 1909-1927. https://doi. org/10.1177/1077801218755976

Barrick, K., Krebs, C. P., \& Lindquist, C. H. (2013). Intimate partner violence victimization among undergraduate women at historically black colleges and universities (HBCUs). Violence Against Women, 19(8), 1014-1033. https://doi.org/10.1177/1077801213499243

Barros, E. N., Silva M. A., Falbo Neto G. H., Lucena S. G., Ponzo, L., \& Pimentel, A. P. (2016). Prevalence and factors associated with intimate partner violence among women in Recife/Pernambuco, Brazil. Ciência \& Saúde Coletiva, 21(2), 591-598. https://doi. org/10.1590/1413-81232015212.10672015

Biroli, F. (2018). Gênero e desigualdades: os limites da democracia no Brasil. Boitempo. 
Borges, J. L., Heine, J. A., \& Dell'Aglio, D. D. (2020). Variables personales y contextuales predictivas de perpetración de violencia en el noviazgo en la adolescencia. Acta Colombiana de Psicología, 23(2), 449-459. https:// doi.org/10.14718/ACP.2020.23.2.16

Brasil. Instituto Nacional de Estudos e Pesquisas Educacionais Anísio Teixeira. (2019). Censo da Educação Superior 2018: notas estatísticas. https://download.inep.gov.br/ educacao_superior/censo_superior/documentos/2019/ censo_da_educacao_superior_2018-notas_estatisticas.pdf

Brock Carneiro, J., Pereira Gomes, N., Matheus Estrela, F., Damasceno Santana, J., Santos Mota, R., \& Lorenzini Erdmann, A. (2017) Violência conjugal: repercussões para mulheres e filhas(os). Escola Anna Nery, 21(4). https://doi.org/10.1590/2177-9465-ean-2016-0346

Buelna, C., Ulloa, E. C., \& Ulibarri, M. D. (2009). Sexual relationship power as a mediator between dating violence and sexually transmitted infections among college women. Journal of Interpersonal Violence, 24(8), 1338-1357. https://doi.org/10.1177/0886260508322193

Buhi, E. R., Clayton, H., \& Surrency, H. H. (2009). Stalking victimization among college women and subsequent help-seeking behaviors. Journal of American College Health, 57(4), 419-426. https://doi.org/10.3200/ JACH.57.4.419-426

Carey, K. B., Durney, S. E., Shepardson, R. L., \& Carey, M. P. (2015). Incapacitated and forcible rape of college women: Prevalence across the first year. Journal of Adolescent Health, 56(6), 678-680. https://doi. org/10.1016/j.jadohealth.2015.02.018

Crenshaw, K. (2002). Documento para o encontro de especialistas em aspectos da discriminação racial relativos ao gênero. Revista Estudos Feministas, 10(1), 171-188. https://doi.org/10.1590/S0104-026X2002000100011

Damra, J. K., \& Abujilban, S. (2018). Violence against women and its consequences on women's reproductive health and depression: A Jordanian sample. Journal of Interpersonal Violence, 36(5-6), 1-17. https://doi. org/10.1177/0886260518770649

Dixon, K. J., Edwards, K. M., \& Gidycz, C. A. (2015). The association of investment model variables and dyadic patterns of physical partner violence: A study of college women. Journal of Interpersonal Violence, 31(16), 2751-2770. https://doi.org/10.1177/0886260515580364

Domínguez Fernández, M., Martínez Silva, I. M., VázquezPortomeñe, F., \& Rodríguez Calvo, M. S. (2017). Características y consecuencias de la violencia de género: estudio de casos confirmados por sentencia judicial. Revista Española de Medicina Legal, 43(3), 115-122. https://doi.org/10.1016/j.reml.2017.05.001

Enríquez-Canto, Y., Ortiz-Montalvo, Y. J., Ortiz-Romaní, K. J., \& Díaz-Gervasi, G. M. (2020). Ecological analysis of intimate partner sexual violence in Peruvian women. Acta Colombiana de Psicología, 23(1), 272-286. http:// doi.org/10.14718/ACP.2020.23.1.13

Fontenot, H. B., \& Fantasia, H. C. (2010). Unwanted sex. The Nurse Practitioner, 35(10), 49-53. https://doi. org/10.1097/01.npr.0000388209.08734.81

Herrera Paredes, J. M. \& Arena Ventura, C. A. (2010). Consumo de alcohol y violencia doméstica contra las mujeres: un estudio con estudiantes universitarias de México. Revista Latino-Americana de Enfermagem, 18, 557-64. https://doi.org/10.1590/ S0104-11692010000700011

Hirigoyen, M. F. (2003). Acoso moral contextos, diferencias, consecuencias y medidas. Norte de Salud Mental, 5(18), 39-47. https://dialnet.unirioja.es/descarga/articu lo/4830119.pdf

Hossain, M. B., Memiah, P., \& Adeynka, A. (2014). Are female college students who are diagnosed with depression at greater risk of experiencing sexual violence on college campus? Journal of Health Care for the Poor and Underserved, 25(3), 1341-1359. https://doi.org/10.1353/ hpu.2014.0146 
Kachaeva, M., \& Shport, S. (2017). Psychological and psychiatric consequences of violence against Women. European Psychiatry, 41, Article S904. https://doi. org/10.1016/j.eurpsy.2017.01.1851

Katz, J., \& Rich, H. (2015). Partner covictimization and post-breakup stalking, pursuit, and violence: A retrospective study of college women. Journal of Family Violence, 30(2), 189-199. https://doi:10.1007/s10896-014-9665-7

Kavak, F., Aktürk, Ü., Özdemir, A., \& Gültekin, A. (2018). The relationship between domestic violence against women and suicide risk. Archives of Psychiatric Nursing, 32(4), 574-579. https://doi.org/10.1016/j.apnu.2018.03.016

Kotan, Z., Kotan, V. O., Yalvaç, H. D., \& Demir, S. (2020). Association of domestic violence against women with sociodemographic factors, clinical features, and dissociative symptoms in patients who receive services from psychiatric outpatient units in Turkey. Journal of Interpersonal Violence, 35(15-16), 2711-2731. https:// doi.org/10.1177/0886260517703372

Kouta, C., Tolma, E. L., \& Pavlou, S. E. (2013). Date rape among Cypriot female college students: An explorative study. Global Health Promotion, 20(3), 38-46. https:// doi.org/10.1177/1757975913499033

Krebs, C. P., Lindquist, C. H., Warner, T. D., Fisher, B. S., \& Martin, S. L. (2009). College women's experiences with physically forced, alcohol- or other drug-enabled, and drug-facilitated sexual assault before and since entering college. Journal of American College Health, 57(6), 639-649. https://doi.org/10.3200/JACH.57.6.639-649

Leite, F. M. C., Santos, D. F., Castro, D. S., Netto, L. A., Moura, M. A. V., \& Lima, E. F. A. (2017). Percepção de mulheres acerca da violência vivenciada. Revista de Pesquisa: Cuidado é Fundamental Online, 9(1), 193199. http://www.index-f.com/pesquisa/2017/91193.php

Liberati, A., Altman, D. G., Tetzlaff, J., Mulrow, C., Gøtzsche, P. C., Ioannidis, J. P. A., Clarke, M., Devereaux, P. J., Kleijnen, J., \& Moher D. (2009). The PRISMA Statement for Reporting Systematic Reviews and Meta-Analyses of Studies That Evaluate Health Care Interventions: Explanation and Elaboration. PLoS Med, 6(7): e1000100. https://doi.org/10.1371/journal. pmed. 1000100

Lindquist, C. H., Barrick, K., Krebs, C., Crosby, C. M., Lockard, A. J., \& Sanders-Phillips, K. (2013). The context and consequences of sexual assault among undergraduate women at historically black colleges and universities (HBCUs). Journal of Interpersonal Violence, 28(12), 2437-2461. https://doi.org/10.1177/0886260513479032

López, O. C. (2017). La violencia económica y/o patrimonial contra las mujeres en el ámbito familiar. Persona y Familia, 1(6), 39-58. https://doi.org/10.33539/peryfa.2017.n6.468

McDaniel, A. (2018). Women's rising share of tertiary enrollment: A cross-national analysis. FIRE: Forum for International Research in Education, 1(2). https://doi. org/10.18275/fire201401021016

Melnyk, B. M. \& Fineout-Overholt, E. (2005). Making the case for evidence based practice. Em: B. M. Melnyk, \& E. Fineout-Overholt (orgs.). Evidencebased practice in nursing \& healthcare. A guide to best practice (pp. 3-24). Lippincot Williams \& Wilkins.

Messman-Moore, T. L., Ward, R. M., \& Brown, A. L. (2009). Substance Use and PTSD symptoms impact the likelihood of rape and revictimization in college women. Journal of Interpersonal Violence, 24(3), 499-521. https://doi.org/10.1177/0886260508317199

Messman-Moore, T. L., Ward, R. M., \& DeNardi, K. A. (2013). The impact of sexual enhancement alcohol expectancies and risky behavior on alcohol-involved rape among college women. Violence Against Women, 19(4), 449-464. https://doi.org/10.1177/1077801213487058

Navarro-Mantas, L., \& Velásquez, M. J. (2016). Herramientas para prevenir la violencia de género: implicaciones de un registro diario de situaciones de desigualdad de género. Acta Colombiana de Psicología, 19(2), 139-148. https:// doi.org/10.14718/ACP.2016.19.2.7 
Organización Panamericana de la Salud \& Centers for Disease Control and Prevention. (2014). Violencia contra las mujeres en América Latina y el Caribe: análisis comparativo de datos poblacionales de 12 países. https://www3.paho.org/hq/in dex.php?option $=$ com_docman\&view $=$ download\&category_slug=violencia-5197\&alias $=24353-$ vio lencia-contra-mujeres-america-latina-caribe-ana lisis-comparativo-datos-poblacionales-12-pai ses-353\&Itemid $=270 \&$ lang $=$ en

Orozco Vargas, A. E., Mercado Monjardín, M. R., García López, G. I., Venebra Muñoz, A., \& Aguilera Reyes, U. (2021). Creencias sobre la violencia y sus efectos en la prevalencia de la violencia en el noviazgo. Acta Colombiana de Psicología, 24(1), 154-166. https://www. doi.org/10.14718/ACP.2021.24.1.14

Pinto, E. J. S., Amorim, V. G., \& Carvalho, M. E. P. (2016). Entre discriminação explícita e velada: experiências de alunas de física na educação superior. Diversidade e Educação, 4(8), 13-32. https://seer.furg.br/divedu/ article/view/6737/4422

Ricoldi, A., \& Artes, A. (2016). Mulheres no ensino superior brasileiro: espaço garantido e novos desafios. ex сеquo, 33, 149-161. https://exaequo.apem-estudos. org/files/2016-07/10_MULHERES_NO_ENSINO_ SUPERIOR_BRASILEIRO.pdf

Saffioti, H. I. B. (2001). Contribuições feministas para o estudo da violência de gênero. Cadernos Pagu, 16, 115136. https://periodicos.sbu.unicamp.br/ojs/index.php/ cadpagu/article/view/8644541

Saffioti, H. I. B. (2015). Gênero, patriarcado, violência. Expressão Popular.

Sandberg, D. A., Valdez, C. E., Engle, J. L., \& Menghrajani, E. (2019). Attachment anxiety as a risk factor for subsequent intimate partner violence victimization: A 6-month prospective study among college women. Journal of Interpersonal Violence, 34(7), 1410-1427. https://doi. org/10.1177/0886260516651314
Santos, H. C. C., Ramos, E. S., \& Espínola, M. R. B. (2015). Rompendo barreiras, conquistando espaços: o movimento feminista no combate às desigualdades à luz da constituição federal de 1988. Revista Dat@venia,7(1), 158-170. http://revista.uepb.edu.br/index.php/datavenia/ article/view/3624-10886-1/2053

Schry, A. R., \& White, S. W. (2013). Sexual assertiveness mediates the effect of social interaction anxiety on sexual victimization risk among college women. Behavior Therapy, 44(1), 125-136. https://doi.org/10.1016/j.beth.2012.09.001

Scott, J. (1995). Gênero: uma categoria útil de análise histórica. Educação \& Realidade, 20(2), 71-99. https://www.seer.ufrgs.br/educacaoerealidade/article/ viewFile/71721/40667

Souza Santos, M., \& Jaeger, F. P. (2018). "Até hoje não sei o que é a palavra amor!": o impacto do abuso sexual em mulheres. DiÁLOGO, 37, 9-20. http://dx.doi. org/10.18316/dialogo.v0i37.3430

Sutherland, M. A., Fantasia, H. C., \& Fontenot, H. (2015). Reproductive coercion and partner violence among college women. Journal of Obstetric, Gynecologic \& Neonatal Nursing, 44(2), 218-227. https://doi. org/10.1111/1552-6909.12550

Sutherland, M. A., Fantasia, H. C., \& Hutchinson, M. K. (2016). Screening for intimate partner and sexual violence in college women: Missed opportunities. Women's Health Issues, 26(2), 217-224. https://doi.org/10.1016/j. whi.2015.07.008

Tetikcok, R., Ozer, E., Cakir, L., Enginyurt, O., İscanli, M. D., Cankaya, S., \& Ozer, F. (2016). Violence towards women is a public health problem. Journal of Forensic and Legal Medicine, 44, 150-157. https://doi.org/10.1016/j. jflm.2016.10.009

Tora, A. (2013). Assessment of sexual violence against female students in Wolaita Sodo University, Southern Ethiopia. Journal of Interpersonal Violence, 28(11), 2351-2367. https://doi.org/10.1177/0886260512475316 
Whittemore, R., \& Knafl, K. (2005). The integrative review: Updated methodology. Journal of Advanced Nursing, 52(5), 546-553. https://doi.org/10.1111/j.1365 $-2648.2005 .03621 . \mathrm{x}$

Wigderson, S., \& Katz, J. (2015). Feminine ideology and sexual assault: Are more traditional college women at greater risk? Violence Against Women, 21(5), 616-631. https://doi.org/10.1177/1077801215573333
Yamakawa, E. K., Kubota, F. I., Beuren, F. H., Scalvenzi, L., $\&$ Cauchick Miguel, P. A. (2014). Comparing the bibliographic management softwares: Mendeley, EndNote and Zotero. Transinformação, 26(2), 167-176. https://doi. org/10.1590/0103-37862014000200006

Yoon, E., Funk, R. S., \& Kropf, N. P. (2010). Sexual harassment experiences and their psychological correlates among a diverse sample of college women. Affilia, 25(1), 8-18. https://doi.org/10.1177/0886109909354979 